\title{
Integrating mental healthcare in primary care in Singapore
}

\author{
Nicole Pei Ching Ooi ${ }^{1}$ MSci, Claudia Zhi Ge Neo ${ }^{1}{ }_{B S c}$, Rebecca Kian Shyan $\underline{\text { Chong }}{ }^{1}$ MSc
}

Management of mental health conditions can be both resource-intensive and costly. The rigour of obtaining appointments and the long waiting time at tertiary hospitals, coupled with the need to obtain leave from work form a significant financial and logistical burden on patients for the treatment of mental health conditions. The alternative-more flexible private practice appointments - comes with a high consultation fee. The direct costs (e.g. medication and consultation fees) are eclipsed by the indirect costs (e.g. loss of wages), with over $80 \%$ of the annual total cost attributed to indirect cost. ${ }^{1}$ Moreover, patients with mental health conditions often have comorbid physical conditions, ${ }^{2}$ and would benefit from holistic co-management of these conditions.

The World Health Organization asserted that integrating mental health into primary care settings would produce clear benefits, such as increased access and affordability. ${ }^{3}$ Singapore has taken steps to integrate mental health services into primary care.

Singapore's National Mental Health Blueprint was initiated in 2007 to improve mental health services. It was centred around integrating mental health services, improving mental health literacy, and developing workforce and research capabilities in communitybased services. Consequently, the 2012 Community Mental Health $(\mathrm{CMH})$ Masterplan proposed the need to lend importance to treating mental health conditions within the community, focusing on early detection and patients with stable, chronic mental health conditions.

In line with these plans is the Ministry of Health's healthcare vision for "One Singaporean, One Family Doctor". It focuses on each Singaporean being in a long-term partnership with a family doctor, and emphasises preventative and community care. This vision enables the primary care ecosystem to integrate mental health and physical health through increased capability and capacity of care access.

Capability and capacity alone are insufficient. The direct and indirect costs of mental healthcare are high, with conditions such as depressive disorder costing patients over USD7,500 (SGD10,000) per year in 2008. ${ }^{1}$ The plans highlighted in the CMH Masterplan would require improving the affordability of mental health services to ensure these are accessible to all. Singapore operates on a copayment model: a combination of government subsidies, patient's out-of-pocket expenditure, and mandatory national savings such as the MediSave Scheme, ${ }^{4}$ which helps all individuals save a portion of their pension for medical expenses. Subsidies are disbursed through schemes such as the Community Health Assist Scheme (CHAS) and the Chronic Disease Management Programme (CDMP). ${ }^{5}$ Mental health conditions with treatments that are covered by these schemes in primary care settings include schizophrenia, major depression, bipolar disorder and anxiety.

There are 2 main ways patients can access mental health services in primary care, via private general practitioner (GP) clinics or publicly funded polyclinics (Fig. 1).

The first way patients can access mental healthcare is through the Mental Health GP Partnership Programme (MHGPP). Piloted in 2003 by the Institute of Mental Health, the MHGPP's main goal was to identify, train and collaborate with GPs. The GP partners assisted in managing patients with stable mental health conditions whose diagnoses were covered under the CDMP Mental Illness scheme, such as depression, anxiety and schizophrenia.

During the pilot process, key issues were identified, such as GPs' lack of confidence in treating mental health conditions and the affordability of psychotropic drugs. ${ }^{6}$ These issues have since been addressed through refresher sessions carried out for GP partners and centralised drug support arrangements for affordability. ${ }^{7}$ GPs are also invited to attend regular case discussions and engagement sessions held by the mental health services housed in tertiary care facilities.

Since then, the focus of the MHGPP model of care has evolved. Currently, GPs not only manage stable patients right-sited from hospitals but also act as gatekeepers to other mental health services. They can consult new walk-in cases, manage patients within their scope of practice, and refer any patients with severe symptoms or conditions not covered by the MHGPP to a partnered

\footnotetext{
${ }^{1}$ Caregiving and Community Mental Health Division, Agency for Integrated Care, Singapore

Correspondence: Claudia Zhi Ge Neo, Caregiving and Community Mental Health Division, Agency for Integrated Care, 5 Maxwell Road, \#10-00 Tower Block, MND Complex, Singapore 069110.

Email: claudia.neo@aic.sg
} 


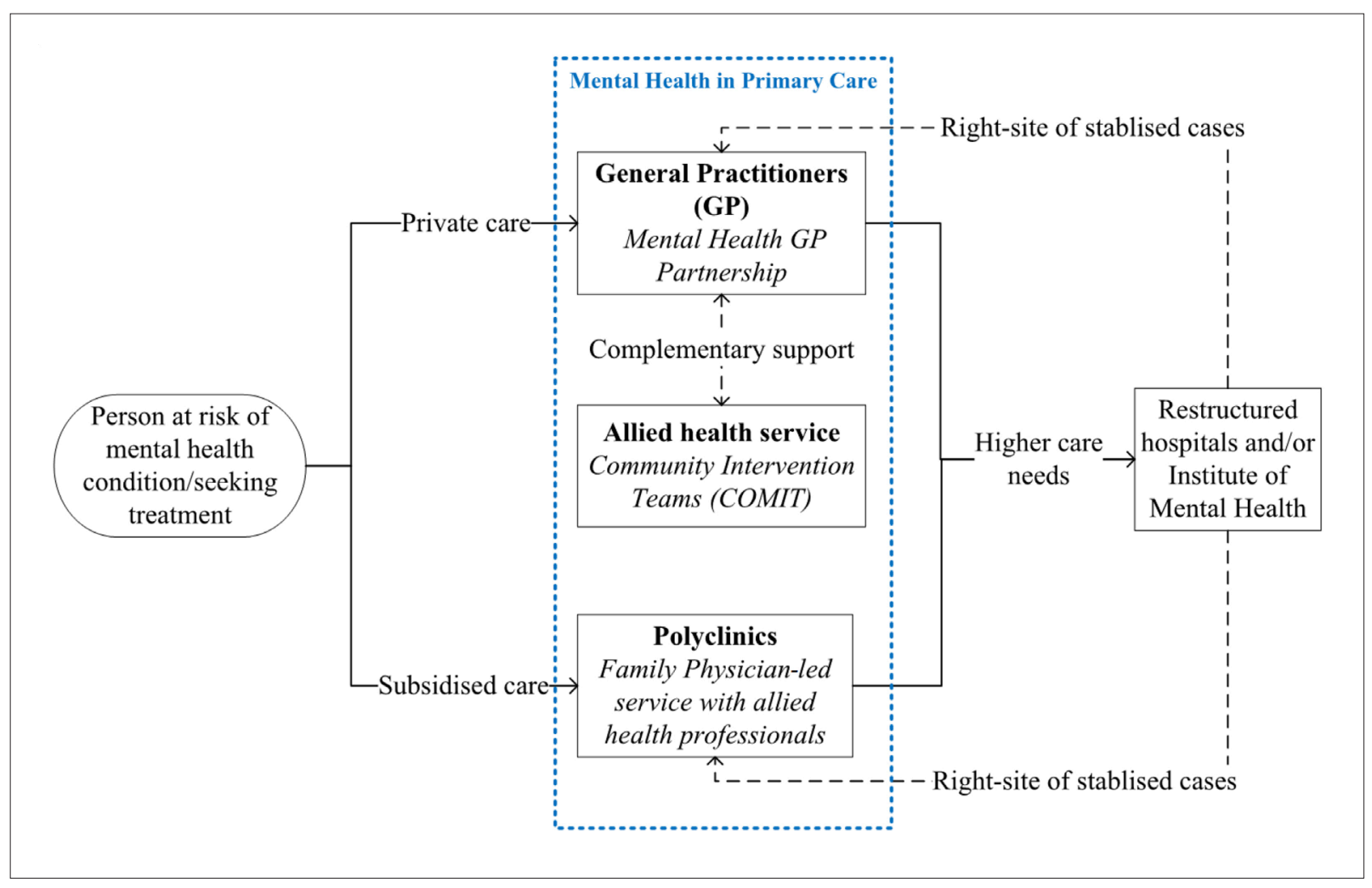

Fig. 1. Mental health services in the primary care setting.

hospital for follow-up. Additionally, the MHGPP has scaled up to affiliate with all public acute hospitals. In 2010, the MHGPP included 40 GPs, with 200 patients successfully right-sited from public hospitals. In 2021, there are now over 200 GPs and over 1,500 patients seen annually, identified through early detection and diagnosis by GPs.

The MHGPP model of care does not operate in isolation. Following the CMH Masterplan in 2012, the Community Intervention Team (COMIT) was developed as a complementary service within the primary care ecosystem. COMIT is an allied health-led team embedded in the community, which offers services to complement the pharmacological interventions provided by GPs. The ecosystem allows for constant information flow and a feedback loop between COMIT and GPs, with GPs able to receive updates on the patient's care from allied health professionals. Besides supporting psychological interventions, COMIT also provides social services, which help mitigate family stressors contributing to mental health issues.

The second avenue for patients to access mental health services in primary care is through polyclinics. Unlike GP clinics that are standalone practices, polyclinics house multiple services such as outpatient medical care and health screenings under one roof. Additionally, unlike GPs in the MHGPP who attend short clinical attachments or refresher courses, family physicians at polyclinics attend clinical attachments, co-consultation with psychiatrists, and monthly multidisciplinary case discussions. Similar to GPs, polyclinics serve a gatekeeping function by referring patients to hospitals for follow-ups. Polyclinics serve an additional function for patients requiring government subsidies, as assessment and referral from a polyclinic is the only route for eligibility to receive subsidised mental healthcare in hospitals. For patients not requiring hospital referrals, doctors in the polyclinics could also refer patients to in-house psychological services.

Polyclinics' mental health capability and capacity have increased over the years. Family physicians and allied health teams provide assessment, diagnosis and management of patients with mild to moderate mental health conditions. Psychological services were uncommon in polyclinic settings before the $\mathrm{CMH}$ Masterplan in 2012. In an effort to improve access, the $\mathrm{CMH}$ Masterplan was enhanced in 2017 to increase capabilities of polyclinics further. The plan: for 1 in 2 polyclinics to have mental health clinics by 2021 . Today, Singapore has achieved this target, with 16 out of 20 polyclinics providing mental health and/or dementia care to over 9,500 patients to date. This demonstrates 
primary care's role in early identification that helps relieve strain on hospital resources and ensure better utilisation of healthcare resources.

Despite the strides that have been made to integrate mental healthcare into primary care, barriers to success still exist. Singapore aims to further improve access to mental healthcare in the coming years. Apart from planning, there is a need to shape an open society that is accepting of mental health conditions, to allow those who require assistance to access services without stigma. There is still prevailing stigmatisation of people with mental health conditions in Singapore, evident through negative images portrayed in the media and social rejection. There appears to be a lack of awareness on some mental health conditions such as obsessivecompulsive disorder $(28.7 \%)$ compared to depression $(55.2 \%) .{ }^{8}$ Public education via campaigns to debunk myths or misconceptions, and increase awareness of mental health is a preliminary step that has seen short-term success. ${ }^{9}$ In 2018, Singapore's National Council of Social Services launched the "Beyond the Label" movement-a 5-year effort to address stigma and go beyond a person's diagnosis. However, it is necessary to examine in detail the effectiveness of these campaigns, for enhancement to ensure that further large-scale campaigns can meet the goals of long-term anti-stigma efforts. There is a lack of peer-reviewed research regarding anti-stigma work in Singapore, and encouraging structured research and publication would allow for evaluations and improvements for future campaigns. ${ }^{10}$

Reviews of the integrated care landscape are also pertinent for continued improvements. Singapore could look to the Brown Primary Care Initiative, which places the GP at the forefront of patient care, ${ }^{11}$ following the Patient-Centered Medical Home (PCMH) model. This model also suggests that technology can drive improved mental health management in primary care settings. The PCMH model utilises telemedicine to assist GPs in managing their mental health cases. Singapore is already making strides in this direction, and in COVID-19, polyclinic psychological services offered teleconsultations to patients. Singapore is also exploring how telemedicine can help increase the range of conditions that GPs can oversee, as well as to allow for support and a second opinion for GPs seeking advice. With further developments, telemedicine as a whole could provide a new, more accessible channel for those currently unable to receive mental healthcare through traditional services.

Singapore is making strides to integrate mental health into primary care, with multiple access points to mental health services in the community.

\section{REFERENCES}

1. Ho RC, Mak KK, Chua AN, et al. The effect of severity of depressive disorder on economic burden in a university hospital in Singapore. Expert Rev Pharmacoecon Outcomes Res 2013;13:549-59.

2. Goldman LS. Comorbid medical illness in psychiatric patients. Curr Psychiatry Rep 2000;2:256-63.

3. World Health Organization and World Organization of Family Doctors. Integrating mental health into primary care: a global perspective. Geneva 2008.

4. Central Provident Fund Board. MediSave. Available at: https:// www.cpf.gov.sg/Members/Schemes/schemes/healthcare/medisave. Accessed on 4 August 2021.

5. Ministry of Health Singapore. Chronic Disease Management Programme (CDMP). Available at: https://www.moh.gov.sg/policiesand-legislation/chronic-disease-management-programme-(cdmp). Accessed on 4 August 2021.

6. Lum AW, Kwok KW, Chong SA. Providing integrated mental health services in the Singapore primary care setting - the general practitioner psychiatric programme experience. Ann Acad Med Singap 2008;37:128-31.

7. Lum WMA, Chew J, Lim BL. The successful collaboration between psychiatrists, a mental health institution and general practitioners in primary care. Singapore Fam Phys 2010;36:19-21.

8. Chong SA, Abdin E, Picco L, et al. Recognition of mental disorders among a multiracial population in Southeast Asia. BMC Psychiatry 2016;16:121.

9. Thornicroft G, Mehta N, Clement S, et al. Evidence for effective interventions to reduce mental-health-related stigma and discrimination. Lancet 2016;12;387:1123-32.

10. Kuek JH, Chen SY, Chua HC. The Need for Scholarly Evaluation of Programmes Targeting Mental Health Stigma in Singapore. Ann Acad Med Singap 2019;48:330-2.

11. Parker DR, Goldman RE, Brown J, et al. The Brown Primary Care Initiative design for strategies towards patient-centered medical home practice transformation. Primary Health Care 2014;4:1-7. 